\title{
A Study on Incidence and Risk Factors of Inguinal Hernia in ESI Population
}

\author{
Dr.S.Vijayakumar M.S. ${ }^{1}$, Dr.R.Alagar samy M.S ${ }^{2}$, \\ ${ }^{1}$ Chief civil surgeon, ${ }^{2}$ Senior Resident, Department of General surgery, \\ Government Medical college and ESIC Hospital, Coimbatore, Tamilnadu, India.
}

\begin{abstract}
Background: Inguinal hernia is one of the most common surgical pathologies. Research studies on clinical factors predisposing a person for the development of inguinal hernia, however, remain scarce. Aims and objective: the present study was to evaluate the incidence and risk factors for the development of inguinal hernia in adult males. Material and Methods: The study was done for a period of one year (January 2015 to December 2015) among the people attending surgical outpatient and inpatient department at Government Medical College and ESIC Hospital, coimbatore. Results: The study population included 130 patients with inguinal hernia, $95 \%$ were males and the majority $23 \%$ belonged to 41 to 50 years. Approximately $23.8 \%$ presented with bilateral inguinal hernia at the time of first presentation. The common risk factors found were heavy object lifting and chronic cough and other co morbid illness. Conclusion: The study had shown a slightly high incidence of bilateral inguinal hernia with the commonest risk factors being chronic cough and heavy weight lifting and affecting the productive group of Esi population.
\end{abstract}

Keywords: Inguinal hernia, Risk factors, Bilateral Inguinal hernia, Constipation, Pantaloon hernia

\section{Introduction}

An Inguinal hernia is a protrusion of abdominal cavity and its contents through the inguinal canal. It is very common in men with lifetime risk of $27 \%$ and $3 \%$ for women ${ }^{1}$. Inguinal hernia repair is a commonly performed general surgery procedure in both adults and children with inguinal hernias constituting more than $95 \%$ of all groin hernia repairs. ${ }^{2}$ Inguinal hernias can either be congenital or acquired. The proposed and well known risk factors and causes for inguinal hernias were increased abdominal pressure, pre existing weakness of abdominal muscles, straining during defecation, heavy lifting of weights, obesity, pregnancy etc. Several hypotheses regarding the cause of inguinal hernia have been proposed; however, large-scale data on the occurrence of inguinal hernia may provide further understanding to the patho physiology of inguinal hernia development. This study was undertaken to estimate the incidence, age and gender differences, risk factors associated with the inguinal hernia. Predominantly in male populations, the risk factors that have been found to be associated with inguinal hernia were muscle weakness (previous appendectomy or other abdominal operations), physical stress, increased intra-abdominal pressure (chronic constipation and prostate enlargement),smoking, aging, connective tissue disease, and systemic illnesses. ${ }^{3}$ In females, obesity, pregnancy, and operative procedures have been shown to be risk factors that commonly contribute to the formation of inguinal hernia. this study was done with the intention of exploring the various aspects of Inguinal hernia and its presentations among adult ESI population.

\section{Aims And Objectives}

1. To estimate the incidence of inguinal hernia among different age groups

2. To assess the risk factors influencing the development of inguinal hernia and determine among which factors inguinal hernia prevalence is more common.

\section{Materials And Methods}

This study was conducted as a hospital based observational study in a tertiary care institute among adults attending surgery outpatient and inpatient department from January 2015 to December 2015 . The study participants were interviewed initially using a questionnaire which included the socio demographic details and symptoms of groin swelling. Study participants came to the hospital with complaints of groin swelling with or without pain were interviewed in depth regarding the duration of swelling, family history of inguinal hernia, lifestyle habits, and nature of job, chronic constipation and cough, co morbidities after getting the informed consent. Then the patients with the groin swelling complaints were completely examined. During the clinical examination the patients were explained about the purpose of the examination and privacy and confidentiality were ensured. An inguinal hernia was diagnosed based on the criteria that clinically detectable swelling in the groin or a clearly palpable defect of the abdominal wall in the groin. The most important clinical signs used to detect hernia were reducibility and cough impulse. No attempt was made to distinguish between indirect and 
direct hernias. We studied associations between hernia and a number of other disorders and characteristics, using data from the questionnaire and examination. We specifically asked for chronic constipation, obstructive pulmonary and urinary tract disease, trauma of the inguinal region or lower abdomen, fractures of the pelvis, and family history for inguinal hernia, including gender and relation with the family member in order to determine a familial tendency. Furthermore, time of exposure of both present and past work activity was recorded.

\section{Results}

Our study included 130 patients presenting with symptomatic or minimally symptomatic inguinal hernia. Among 130 persons, 125 were males and 5 were females(table 1:2). The age-distributed incidence rates of inguinal hernia among the study population was $6 \%, 3 \%, 9 \%, 17.6 \%, 23 \%, 18.4 \%, 13 \%, 9 \%$,in less than 10 years, 11 to 20 years, 21 to 30 years, 31 to 40 years, 41 to 50,51 to 60 years ,61 to 70 years and 70 years above respectively(Table1:1). As far as the presentation of inguinal hernia was concerned $68(52.3 \%)$ had right sided hernia and $31(23.8 \%)$ had left sided hernia. Bilateral inguinal hernia was present among $31(23.8 \%)$ of the study population (Table1:3). Around7 (5.38\%) cases had family history of hernias, $5(3.84 \%)$ cases with bowel disturbances like chronic constipation, $21(16.15 \%)$ cases had bladder disturbances like frequent urination, 14 $(10.76 \%)$ cases had history of chronic cough. While assessing their lifestyle habits $22(16.92 \%)$ cases were chronic smokers and $7(5.38 \%)$ cases were alcoholics. By assessing the occupational status of all patients, 33 $(25.38 \%)$ cases were found to be having positive history of heavy object lifting. As mentioned above, few cases were presented with other co morbidities.

Table 1:1 Age wise distribution of inguinal hernia

\begin{tabular}{|l|l|l|l|}
\hline Sl.no & \multicolumn{1}{|c|}{ Age group } & No. of patients & $\%$ of patients \\
\hline 1. & $1-10$ & 8 & 6 \\
\hline 2. & $11-20$ & 4 & 3 \\
\hline 3. & $21-30$ & 12 & 9 \\
\hline 4. & $31-40$ & 23 & 17.6 \\
\hline 5. & $41-50$ & 30 & 23 \\
\hline 6. & $51-60$ & 24 & 18.4 \\
\hline 7. & $61-70$ & 17 & 13 \\
\hline 8. & Above 70 & 12 & 9 \\
\hline
\end{tabular}

Table 1:2 Sex wise distribution of inguinal hernia

\begin{tabular}{|l|l|l|l|}
\hline Sl.no & Sex wise & No of patients & $\%$ of patients \\
\hline 1. & Male & 125 & 95 \\
\hline 2. & Female & 5 & 5 \\
\hline
\end{tabular}

Table 1:3 Side wise distribution of inguinal hernia

\begin{tabular}{|l|l|l|l|}
\hline Sl.no & Side wise & No of patients & $\%$ of patients \\
\hline 1. & Right & 68 & 52.3 \\
\hline 2. & Left & 31 & 23.8 \\
\hline 3. & Bilateral & 31 & 23.8 \\
\hline
\end{tabular}

Table 1:4 Type of inguinal hernia

\begin{tabular}{|l|l|l|l|}
\hline Sl.no & Type of Hernia & No of patients & $\%$ of patients \\
\hline 1. & Direct inguinal Hernia & 80 & 61.55 \\
\hline 2. & Indirect inguinal Hernia & 37 & 28.46 \\
\hline 3. & Pantaloon inguinal Hernia & 5 & 3.84 \\
\hline 4. & Congenital inguinal Hernia & 8 & 6.15 \\
\hline
\end{tabular}

Table1:5 Risk factors of inguinal hernia

\begin{tabular}{|l|l|l|l|}
\hline Sl.no & Risk factors & No of patients & \% of patients \\
\hline 1. & Family history & 7 & 5.38 \\
\hline 2. & Bowel disturbances & 5 & 3.84 \\
\hline 3. & Chronic cough & 14 & 10.76 \\
\hline 4. & Bladder disturbances & 21 & 16.15 \\
\hline 5. & Smoking habits & 22 & 16.92 \\
\hline 6. & Alcohol drinkers & 7 & 5.38 \\
\hline 7. & Heavy object lifting & 33 & 25.38 \\
\hline 8. & Coexisting co-morbidities & 21 & 16.15 \\
\hline
\end{tabular}

\section{Discussion}

Our study had explored the evidence for age- and gender distribution of inguinal hernias. The agedistributed prevalence rates shows that inguinal hernia prevalence was peaking at adult age group of $41-50$ which constituted almost $23 \%$ of inguinal hernias in this study whereas Indrani Basu et al study showed that the 
peak incidence of inguinal hernia was 42 to 57 years $^{4}$. It is relatively less common in adolescent age groups. This evidence was not supported by many studies . But in some studies it is shown that age distribution is bimodal peaking at early childhood and old age ${ }^{5}$. As shown in the results that out of 130 cases, 125 were males and 5 were females which showed the male to female ratio of 25:1. This might be due to anatomical difference between males and females. Moreover females seek medical attention for their hernia immediately. Most patients see a physician only after pain and discomfort that limits their activity ${ }^{6}$. It is also shown that majority of patients were found to be presenting with reducible hernias i.e., swellings can be pushed back into the abdomen by lying down or putting manual pressure to $i^{7}$. Our study has studied all potential risk factors as reported in the literature. The main risk factor associated with inguinal hernias was found to be heavy object lifting. The results showed that those who had previous history of heavy object lifting for $\geq 10$ yrs were more prone for developing inguinal hernias. In these 130 cases, about half of the men were found to be doing strenuous jobs. This is because majority of patients attending the hospital were low socio-economic industrial working people from surrounding areas. Their main occupation is industrial works etc., which involves increased physical activity which in turn accounts for increased abdominal pressure resulting in developing inguinal hernias. For these men, a durable repair is relatively important to prevent recurrence. The other contributing factors were found to be straining during urination and defecation \&chronic cough in case of TB. These factors increase abdominal pressure resulting in developing inguinal hernias. In chronic smokers (22\%), muscles get weakened which may further increase risk for developing inguinal hernias. The risk factors found out in this study were similar to the study done in USA which showed that the inguinal hernia was associated with older age, obesity, greater height, chronic cough, rural residence ${ }^{10}$. This was supported by many other studies like Lau $\mathrm{H}$ et al and Junge $\mathrm{K}$ et al, which showed that family history is an important predictor for development of inguinal hernias and as well as recurrent hernia ${ }^{8}$.The other risk factors suggested were Hypertension and Diabetes were present in approximately $11 \%$ and $5 \%$ cases respectively ${ }^{9}$ while chronic cough, chronic constipation, Chronic Diabetes and Prostatic hypertrophy ${ }^{10,11,12}$. Symptoms of prostate enlargement were present in approximately $21 \%$ cases .Any activity or medical problem that increases pressure on the tissue in the belly wall and muscles may lead to a hernia. These include prostatic hypertrophy and anal fissures which causes straining during urination and defecation respectively. Some other studies reported that prostatic hypertrophy and varicosities were associated with hernia.

\section{Conclusion}

This incidence study showed that the age distribution of inguinal hernia repair was peaking at adult age with male preponderance of 25: 1 male to female ratio. Our study elicited that heavy object lifting and chronic cough were major risk factors for inguinal hernia in males. Henceforth, in patients with unexplained lower abdominal pain, the presence of these factors should prompt the physician to consider the diagnosis of inguinal hernia. This information can potentially create a basis for formulating new hypotheses regarding disease cause. It is concluded that these results may be a useful guide for future studies about the incidence of inguinal hernias in the populations as a whole.

\section{Source of Support: Nil}

Conflict of Interest: Nil.

\section{References}

[1]. John T Jenkins, Patrick J O’Dwyer "Inguinal hernias". British Medical Journal. BMJ 336 (7638): Page. $269-272$.

[2]. Ein SH, Njere I, Ein A (2006) Six thousand three hundred sixty-one pediatric inguinal hernias: a 35year review. J PediatrSurg 41: 980-6.

[3]. Rutkow IM (2003) Demographic and socioeconomic aspects of hernia repair in the United States in 2003. SurgClin North Am 83: $1045-51$.

[4]. Indranil Basu, Sudhangshou Sekhar Bhoj, Ananda Kumar Mukhopathyay. Retrospective Study on Prevalence of Primary and Recurrent Inguinal Hernia and its Repairs in Patients Admitted to a Tertiary Care Hospital. Indian Medical Gazette — JUNE 2013. Page $203-213$

[5]. Burcharth J, Pedersen M, Bisgaard T, Pedersen C, Rosenberg J. Nationwide Prevalence of Groin Hernia Repair. PLoS one. 2013;8(1) PLoS One. 2013;8(1):e54367. doi: 10.1371/journal.pone.0054367. Epub 2013 Jan 14.

[6]. Gulzar, M.R., Iqbal, J., Ulhaq, M.I. and Afzal, M. (2007) Darning vs Bassini repair for inguinal hernia-A pro- spective comparative study. Professional Medical Jour- nal, 14, 128-133.

[7]. Zimmermann, L.M. and Amson, B.J. (1967) Anatomy and surgery of hernias, 2nd Edition, Willium and Wilkins, Baltimore.

[8]. Fitzgibbons, R.J., Filipi, C.J. and Thomas, H.Q. (2005) Inguinal hernia. In: Brunicardi, F.C., Andersen, D.K., Bi- lliar, T.R., Dunn, D.L., Hunter, J.G. and Pollock, R.E., Eds., Schwartz's Principles of Surgery, 8th Edition, McGraw- Hill, New York.

[9]. Mukesh Sangwan1, Vijayata Sangwan, Mahender Garg, Parveen Mahendirutta, Uma Garg. Abdominal wall hernia in a rural population in India-Is spectrum changing? - Open journal of epidemiology 2013, 3, page 135 - 138.

[10]. Constance E. Ruhl1 and James E. Everhart. Risk Factors for Inguinal Hernia among Adults in the US Population. American journal of Epidemiology. Am J Epidemiol. 2007; 165 (10). Page 1154 - 1161.

[11]. Lau H., Fang C., Yuen W.K., Patil N.G. — Risk factors for inguinal hernia in adult males: A casecontrol study. Surgery. 141:262266, 2007.

[12]. Junge K., Rosch R., Klinge U., Schwab R., Peiper C., Binnebosel M., et al. — Risk factors related to recurrence in inguinal hernia repair: a retrospective analysis. Hernia. 10:309-315, 2006. 